\title{
LEBESGUE PROPERTY OF CONVEX RISK MEASURES FOR BOUNDED CÀDLÀG PROCESSES*
}

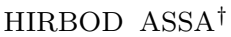

\begin{abstract}
In this paper, we study the Lebesgue property for convex risk measures on the class of bounded càdlàg processes. For that, we characterize the compact subsets of a family of bounded variation processes, which is, of course, the topological dual of the bounded càdlàg processes, in an appropriate topology. We show that the Lebesgue property can be characterized in several equivalent ways.
\end{abstract}

Key words. Convex risk measures, bounded càdlàg processes, Lebesgue property, static risk.

AMS subject classifications. 91B30, 91B16, 60G07, 52A07, 46A20.

1. Introduction. The Fatou property and the Lebesgue property appear in the context of Locally Solid Riesz Spaces. A Locally Solid Riesz Space has the Fatou property if the semi-norms generating its topology are continuous from below and it has the Lebesgue property if the semi-norms are continuous from above (see Aliprantis and Burkinshaw [1]). While these properties are well studied in the context of Locally Solid Riesz Spaces, they enter financial mathematics literature with slightly different definitions. Delbaen [6] introduces the Fatou property for a coherent risk measure as continuity with respect to decreasing sequences. Föllmer and Schied [8] similarly extend the definition of the Fatou property to a convex risk measure. Jouini, Schachermayer and Touzi [10] introduce the Lebesgue property for a convex risk measure as continuity with respect to increasing sequences. Coherent risk measures for finite probability spaces are introduced in Artzner et al. [2] and extended to general probability spaces in Delbaen [6]. In Föllmer and Schied [8], the authors define a more general notion of convex risk measures and extend the representation results of Delbaen [6]. In Cheridito et al. [4,5], the authors study convex risk measures for bounded càdlàg processes. They introduce the Fatou property in this new setting and come up with the same representations results that have been established for convex risk measures on the space of bounded random variables.

In this paper, we extend the definition of the Lebesgue property to the space of bounded càdlàg processes. We characterize the risk measures with the Lebesgue property in several equivalent ways. Our main goal is to find equivalent conditions for the Lebesgue property in terms of conditions that can be readily verified, which easily allows us to identify this property for complicated yet interesting convex risk measures. The Lebesgue property allows us to approximate a convex risk measure for bounded càdlàg process $X$ with the risk associated with a uniformly bounded sequence $X_{n}$ converging to $X$. This is important when we deal with a time discretization of a finite time horizon. This type of approximation can only be carried out using the Fatou property if the approximating processes are decreasing sequences, which cannot always be carried out with time discretization. Having the Lebesgue property, we only need a uniformly bounded sequence of approximating processes. This will be discussed in Section 4. The other important feature of the Lebesgue property is

\footnotetext{
*Received January 4, 2011; accepted for publication March 14, 2012

$\dagger$ Department of Mathematics and Statistics, University of Montreal, Pavillon André-Aisenstadt 2920, chemin de la Tour, bureau 5190 Montreal, Quebec H3T 1J4, Canada (assa@dms.umontreal.ca \& assa.hirbod@gmail.com).
} 
that the maximum is attained in the so-called Fenchel representation of a convex risk measure. This important property can be used in many optimization problems.

The paper is organized as follows. In Section 2, we recall basic definitions and results for convex risk measures of random variables, and for a class of càdlàg processes. In particular, we state two results: one related to the Fatou property for risk measures on the spaces of bounded càdlàg processes, and another one related to the Lebesgue property for risk measures on the space of bounded random variables. We present the main results of the paper in Section 3. We pay particular attention to characterizing relatively compact subsets of a given dual space, and to characterizing the Lebesgue property. Furthermore, we present an extended version of James' Theorem. In Section 4, we give some examples of risk measures with the Lebesgue property.

2. Preliminaries and remarks. Let $(\Omega, \mathcal{F}, \mathbb{P})$ be a standard and atom-less probability space and let $\left(\mathcal{F}_{t}\right)_{0 \leq t \leq T}$ be a filtration with the usual conditions. Furthermore, assume that $L^{1}(\Omega, \mathcal{F})$ has a countable dense subset.

In $[4,5]$ the authors developed the theory of convex risk measures on the space of $\mathcal{R}^{p}$ consisting of stochastic processes on $[0, T]$ that are càdlàg, adapted and such that $X^{*}=\sup _{[0, T]}\left|X_{t}\right| \in L^{p}$, with $1 \leq p \leq \infty$. Note that for any $1 \leq p \leq \infty, \mathcal{R}^{p}$, endowed with the norm $\|X\|_{\mathcal{R}^{p}}=\left\|X^{*}\right\|_{L^{p}}$, is a Banach space.

For $q \in[1, \infty]$, let $\mathcal{A}^{q}$ be the set of all $a=\left(a^{\mathrm{pr}}, a^{\mathrm{op}}\right):[0, T] \times \Omega \rightarrow \mathbb{R}^{2}$ such that $a^{\mathrm{pr}}$ and $a^{\mathrm{op}}$ are right continuous, have finite variation in $L^{q}, a^{\mathrm{pr}}$ is predictable, and $a_{0}^{\mathrm{pr}}=0, a^{\mathrm{op}}$ is optional and purely discontinuous.

Denoting the variation of a function $f:[0, T] \rightarrow \mathbb{R}$ by $\operatorname{Var}(f)$, it follows that $\mathcal{A}^{q}$ is also a Banach space, when equipped with the norm

$$
\|a\|_{\mathcal{A}^{q}}=\|\operatorname{Var}(a)\|_{L^{q}},
$$

where by $\operatorname{Var}(a)$ we mean $\operatorname{Var}\left(a^{\mathrm{pr}}\right)+\operatorname{Var}\left(a^{\mathrm{op}}\right)$. and $\mathcal{R}^{p}$

Furthermore, if $p$ and $q$ satisfy $\frac{1}{p}+\frac{1}{q}=1$, there is a duality relation between $\mathcal{A}^{q}$

$$
\langle X, a\rangle=\mathbb{E}\left[\int_{] 0, T]} X_{t-} d a_{t}^{\mathrm{pr}}+\int_{[0, T]} X_{t} d a_{t}^{\mathrm{op}}\right], \quad(X, a) \in \mathcal{R}^{p} \times \mathcal{A}^{q} .
$$

Note that

$$
|\langle X, a\rangle| \leq\|X\|_{\mathcal{R}^{p}}\|a\|_{\mathcal{A}^{q}} .
$$

The subset $\mathcal{A}_{+}^{q}$ of $\mathcal{A}^{q}$ consisting of $a=\left(a^{p r}, a^{o p}\right)$ with both components non-negative and non-decreasing, will be important in the sequel.

Further, let $\mathcal{D}_{\sigma}$ be the members of $\mathcal{A}_{+}^{1}$ such that

$$
\|a\|_{\mathcal{A}^{1}}=\|\operatorname{Var}(a)\|_{L^{1}}=1 \text {. }
$$

For two random process $X$ and $W$, by $X \leq W$ we mean that there exists a set $\Omega^{\prime}$ of measure one such that

$$
X_{t}(\omega) \leq W_{t}(\omega) \text { for all } t \in[0, T], \omega \in \Omega^{\prime}
$$

Definition 2.1. A convex risk measure $\rho$ on $\mathcal{R}^{\infty}$ is a mapping from $\mathcal{R}^{\infty}$ to $\mathbb{R}$ such that for any $X, W \in \mathcal{R}^{\infty}$ : 
1. $\rho(\lambda X+(1-\lambda) W) \leq \lambda \rho(X)+(1-\lambda) \rho(W)$, for all $0 \leq \lambda \leq 1$.

2. $\rho(X+m)=\rho(X)-m$, for any $m \in \mathbb{R}$.

3. $\rho(X) \geq \rho(W)$, whenever $X \leq W$.

$\rho$ is called a coherent risk measure if in addition

4. $\rho(\lambda X)=\lambda \rho(X)$ for any $\lambda>0$.

In Cheridito et al. [4] the Fatou property is defined as

Definition 2.2. A convex risk measure $\rho$ on $\mathcal{R}^{\infty}$ has Fatou property if for any bounded sequence $\left\{X_{n}\right\}_{n \in \mathbb{N}} \subseteq \mathcal{R}^{\infty}$, for which there exists $X \in \mathcal{R}^{\infty}$ so that $\left(X_{n}-\right.$ $X)^{*} \stackrel{\mathbb{P}}{\rightarrow} 0$, we have $\rho(X) \leq \liminf \rho\left(X_{n}\right)$.

In Cheridito et al [4] it is proven that a risk measure $\rho$ has the Fatou property if and only if it has the following representation

$$
\rho(X)=\sup _{a \in \mathcal{D}_{\sigma}}\{\langle-X, a\rangle-\gamma(a)\}, X \in \mathcal{R}^{\infty},
$$

for some penalty function $\gamma$. Recall that $\gamma$ is a penalty function if $\gamma: \mathcal{D}_{\sigma} \rightarrow(-\infty,+\infty]$ is such that $-\infty<\inf _{a \in \mathcal{D}_{\sigma}} \gamma(a)<\infty$. by

Moreover, in each case, the conjugate function $\rho^{*}$, restricted to $\mathcal{D}_{\sigma}$, and defined

$$
\rho^{*}(a)=\sup _{X \in \mathcal{R}^{\infty}}\{\langle-X, a\rangle-\rho(X)\},
$$

is a penalty function which is smaller than $\gamma$ and $\gamma$ can be replaced by $\rho^{*}$ in (2.2).

Remark 2.1. As mentioned in [4], $\rho^{*}$ restricted to $\mathcal{D}_{\sigma}$ equals $\rho^{\#}$ defined as follows:

$$
\rho^{\#}(a):=\sup _{X \in \mathcal{A}_{\rho}}\langle-X, a\rangle,
$$

where $\mathcal{A}_{\rho}:=\left\{X \in \mathcal{R}^{\infty} \mid \rho(X) \leq 0\right\}$ is the acceptance set of $\rho$. This implies that

$$
\rho(X)=\sup _{a \in \mathcal{D}_{\sigma}}\left\{\langle-X, a\rangle-\rho^{\#}(a)\right\}, X \in \mathcal{R}^{\infty} .
$$

The following corollary is also taken from [4].

Corollary 2.1. A coherent risk measure $\rho$ on $\mathcal{R}^{\infty}$ has Fatou property if and only if there exists a subset $\mathcal{Q}$ of $\mathcal{D}_{\sigma}$ such that

$$
\rho(X)=\sup _{a \in \mathcal{Q}}\langle-X, a\rangle .
$$

In general $\mathcal{Q}$ is not unique. An appropriate choice for $\mathcal{Q}$ is $\operatorname{dom}\left(\rho^{*}\right) \cap \mathcal{A}_{+}^{1}=\{a \in$ $\left.\mathcal{A}_{+}^{1} \mid \rho^{*}(a)=0\right\}$.

Next, we propose our definition of the Lebesgue property for risk measures on $\mathcal{R}^{\infty}$, which is an extension of the Lebesgue property on $L^{\infty}$, introduced in [10] 
Definition 2.3. A convex risk measure $\rho$ on $\mathcal{R}^{\infty}$ has Lebesgue property if for any bounded sequence $\left\{X_{n}\right\}_{n \in \mathbb{N}} \subseteq \mathcal{R}^{\infty}$, for which there exists $X \in \mathcal{R}^{\infty}$ so that $\left(X_{n}-\right.$ $X)^{*} \stackrel{\mathbb{P}}{\rightarrow} 0$, we have $\rho(X)=\lim \rho\left(X_{n}\right)$.

Define

$$
\hat{\mathcal{R}}^{p}=\left\{\begin{array}{l|l}
X:[0, T] \times \Omega \rightarrow \mathbb{R} \mid \begin{array}{l}
X \text { is càdlàg } \\
X^{*} \in L^{p}
\end{array}
\end{array}\right\}
$$

and

$$
\hat{\mathcal{A}}^{q}=\left\{\begin{array}{l|l}
a:[0, T] \times \Omega \rightarrow \mathbb{R}^{2} & \begin{array}{l}
a=\left(a^{\mathrm{l}}, a^{\mathrm{r}}\right), a_{0}^{l}=0 \\
a^{\mathrm{l}}, a^{\mathrm{r}} \text { measurable } \\
\text { finite variation } \\
\text { and right continuous } \\
\operatorname{Var}\left(a^{\mathrm{l}}\right)+\operatorname{Var}\left(a^{\mathrm{r}}\right) \in L^{q}
\end{array}
\end{array}\right\} .
$$

Furthermore, extend the duality relation (2.1) by setting

$$
\langle X, a\rangle=\mathbb{E}\left[\int_{] 0, T]} X_{t-} d a_{t}^{1}+\int_{[0, T]} X_{t} d a_{t}^{\mathrm{r}}\right], \quad(X, a) \in \hat{\mathcal{R}}^{p} \times \hat{\mathcal{A}}^{q}
$$

REMARK 2.2. Following discussions in Remark 3.7. in [4] we know that there exist a continuous mapping $\Pi^{*}:\left(\hat{\mathcal{A}}^{q}, \sigma\left(\hat{\mathcal{A}}^{q}, \hat{\mathcal{R}}^{p}\right)\right) \rightarrow\left(\mathcal{A}^{q}, \sigma\left(\mathcal{A}^{q}, \mathcal{R}^{p}\right)\right)$ such that

$$
\langle X, a\rangle=\left\langle X, \Pi^{*}(a)\right\rangle, \quad \forall(X, a) \in \mathcal{R}^{p} \times \hat{\mathcal{A}}^{q} .
$$

REMARK 2.3. Let $Y \in L^{p}(\Omega, \mathcal{F})$ be a random variable. By Doob's Stopping Theorem it is easy to see that the constant random process ${ }^{1} X_{t}=Y, \forall t \in[0, T]$ is the martingale $M_{t}:=\mathbb{E}\left[Y \mid \mathcal{F}_{t}\right]$. It follows that for every $Y \in L^{p}$ and every $a=\left(a^{p r}, a^{o p}\right) \in$ $\mathcal{A}^{q}$ which is also adapted, one has

$$
\mathbb{E}[\operatorname{Var}(a) Y]=\langle X, a\rangle=\langle M, a\rangle=\left\langle\mathbb{E}\left[Y \mid \mathcal{F}_{t}\right]_{t \in[0, T]}, a\right\rangle .
$$

Throughout this paper we focus our attention to the case $p=\infty$. The case $p<\infty$ can be carried out in an easier fashion, which is left to reader.

Definition 2.4. To every convex risk measure $\rho$ on $\mathcal{R}^{\infty}$, one associates a convex risk measure on $L^{\infty}$, called the static risk,

$$
\bar{\rho}(Y):=\rho\left(\mathbb{E}\left[Y \mid \mathcal{F}_{t}\right]_{0 \leq t \leq T}\right), \quad Y \in L^{\infty},
$$

and a static minimal penalty,

$$
\overline{\rho^{\#}}(f):=\inf _{\left\{a \in \mathcal{D}_{\sigma} \mid \operatorname{Var}(a)=f\right\}} \rho^{\#}(a), \quad f \in D_{\sigma} .
$$

We know from [6] that $\bar{\rho}$ can be represented as

$$
\rho(Y)=\sup _{f \in D_{\sigma}}\left\{\mathbb{E}[-f Y]-\rho^{*}(f)\right\}
$$

\footnotetext{
${ }^{1}$ Indeed its optional projection, see [7]
} 
where $D_{\sigma}:=\left\{f \in L^{1}+\mid \mathbb{E}[f]=1\right\}$. Also it is pointed out in [8] that

$$
\rho^{\#}(f):=\sup _{Y \in \mathcal{A}_{\rho}} E[-f Y]=\rho^{*}(f), \forall f \in D_{\sigma},
$$

where $\mathcal{A}_{\rho}=\left\{Y \in L^{\infty} \mid \rho(Y) \leq 0\right\}$. In the following we see the relation between the static minimal penalty and the minimal static penalty, but before recall that $\Pi^{\mathrm{op}}$ and $\Pi^{\mathrm{pr}}$ denote the optional and predictable projections, respectively, see [7].

TheOREM 2.1. For every risk measure $\rho: \mathcal{R}^{\infty} \rightarrow \mathbb{R}$ the static minimal penalty equals the minimal static penalty i.e.

$$
\overline{\rho^{\#}}=(\bar{\rho})^{\#} \text {. }
$$

Proof. First of all, we show that $\overline{\rho^{\#}}$ is a convex and lower semi-continuous function on $D_{\sigma}$. Let $f, g, h \in D_{\sigma}$ be such that $f=\lambda g+(1-\lambda) h$ for some $\lambda \in(0,1)$. Let $b, c \in \mathcal{D}_{\sigma}$ be such that $\operatorname{Var}(b)=g$ and $\operatorname{Var}(c)=h$. Since $b, c \in \mathcal{D}_{\sigma}$ then $\operatorname{Var}(\lambda b+(1-$ $\lambda) c)=\lambda \operatorname{Var}(b)+(1-\lambda) \operatorname{Var}(c)=f$ which gives $\lambda b+(1-\lambda) c \in\left\{a \in \mathcal{D}_{\sigma} \mid \operatorname{Var}(a)=f\right\}$. Therefore we have

$$
\inf _{\left\{a \in \mathcal{D}_{\sigma} \mid \operatorname{Var}(a)=f\right\}} \rho^{*}(a) \leq \rho^{*}(\lambda b+(1-\lambda) c) \leq \lambda \rho^{*}(b)+(1-\lambda) \rho^{*}(c),
$$

where in the second inequality we use the convexity of $\rho^{*}$. Taking the infimum over all $b, c$ for which $\operatorname{Var}(b)=g$ and $\operatorname{Var}(c)=h$, we have the convexity of $\overline{\rho^{\#}}$.

Let $X \in \mathcal{R}^{\infty}$ and define $\phi(f)=\sup _{\left\{a \in \mathcal{D}_{\sigma} \mid \operatorname{Var}(a)=f\right\}}\langle X, a\rangle$ for all $f \in D_{\sigma}$. We claim that $\phi$ is linear and $\sigma\left(L^{1}, L^{\infty}\right)$-lower semi-continuous on $D_{\sigma}$. One can consider that $X>1$, since otherwise one takes $X+\|X\|_{\mathcal{R}^{\infty}}+1$ instead.

First, let us see that since $X=\Pi^{\mathrm{op}}(X)$ then $\langle X, a\rangle=\left\langle\Pi^{\mathrm{op}}(X), a\right\rangle=\left\langle X, \Pi^{*}(a)\right\rangle$ for all $a \in \hat{\mathcal{D}}_{\sigma}$. This gives that

$$
\phi(X)=\sup _{\left\{a \in \mathcal{D}_{\sigma} \mid \operatorname{Var}(a)=f\right\}}\langle X, a\rangle=\sup _{\left\{a \in \hat{\mathcal{D}}_{\sigma} \mid \operatorname{Var}(a)=f\right\}}\langle X, a\rangle, \forall X \in \mathcal{R}^{\infty} .
$$

Let $0<\epsilon \leq 1$ and define $X^{\epsilon}$ as

$$
X^{\epsilon}:=\min \left\{X, X^{*}-\epsilon\right\} .
$$

It is clear that $X^{\epsilon}$ is a càdlàg process and then in $\hat{\mathcal{R}}^{\infty}$. Also $\left(X^{\epsilon}\right)^{*}=X^{*}-\epsilon$. Let $\theta^{\epsilon}=\inf \left\{t \leq T \mid X^{\epsilon}=X^{*}-\epsilon=\left(X^{\epsilon}\right)^{*}\right\}$. Thus, $\theta^{\epsilon}$ is a random time and not necessarily a stopping time. It is also clear that $X_{\theta^{\epsilon}}^{\epsilon}=\left(X^{\epsilon}\right)^{*}=X^{*}-\epsilon$. Let $f \in D_{\sigma}$, and define $\phi^{\epsilon}(f)=\sup _{\left\{a \in \hat{\mathcal{A}}^{1} \mid \operatorname{Var}(a)=f\right\}}\left\langle X^{\epsilon}, a\right\rangle$. Let $a^{\epsilon}=\left(0,1_{\left[\theta^{\epsilon}, T\right]} f\right)$. It is clear that $a^{\epsilon} \in \hat{\mathcal{D}}_{\sigma}$ and $\operatorname{Var}\left(a^{\epsilon}\right)=f$.

From the definition of $\phi, X^{\epsilon}$ and $\theta^{\epsilon}$ we have

$$
\phi^{\epsilon}(f) \geq\left\langle X^{\epsilon}, a^{\epsilon}\right\rangle=\mathbb{E}\left[X_{\theta^{\epsilon}}^{\epsilon} f\right]=\mathbb{E}\left[\left(X^{*}-\epsilon\right) f\right] .
$$

On the other hand it is clear that

$$
\phi^{\epsilon}(f) \leq \mathbb{E}\left[\left(X^{\epsilon}\right)^{*} f\right]=\mathbb{E}\left[\left(X^{*}-\epsilon\right) f\right] .
$$


This inequality along with the previous one yield $\phi^{\epsilon}(f)=\mathbb{E}\left[\left(X^{*}-\epsilon\right) f\right]$. By (2.12) we have

$$
\begin{aligned}
\left|\phi(f)-\phi^{\epsilon}(f)\right| & =\left|\sup _{\left\{a \in \hat{\mathcal{D}}_{\sigma} \mid \operatorname{Var}(a)=f\right\}}\langle X, a\rangle-\sup _{\left\{a \in \hat{\mathcal{D}}_{\sigma} \mid \operatorname{Var}(a)=f\right\}}\left\langle X^{\epsilon}, a\right\rangle\right| \\
& \leq \sup _{\left\{a \in \hat{\mathcal{D}}_{\sigma} \mid \operatorname{Var}(a)=f\right\}}\left|\left\langle X^{\epsilon}-X, a\right\rangle\right| \\
& \leq \epsilon \mathbb{E}[f] \stackrel{\epsilon \rightarrow 0}{\longrightarrow} 0
\end{aligned}
$$

This shows that $\phi(f)=\mathbb{E}\left[f X^{*}\right]$ on $D_{\sigma}$. In general when $X>1$ is not necessarily true, it is easy to see that $\phi(f)=\mathbb{E}\left[\left(\left(X+X^{*}\right)^{*}-X^{*}\right) f\right]$, which shows that $\phi$ is linear as well as $\sigma\left(L^{1}, L^{\infty}\right)$-lower semi continuous. Now observe that since by Theorem 3.2 $\left\{a \in \mathcal{D}_{\sigma} \mid \operatorname{Var}(a)=f\right\}$ is a convex compact set in the locally convex topological space $\left(\mathcal{A}^{1}, \sigma\left(\mathcal{A}^{1}, \mathcal{R}^{\infty}\right)\right)$, a Minimax Theorem mentioned in [6] yields

$$
\overline{\rho^{\#}}(f)=\inf _{\operatorname{Var}(a)=f} \rho^{\#}(a)=\inf _{\{\operatorname{Var}(a)=f\}} \sup _{\{\rho(X) \leq 0\}}\langle-X, a\rangle=\sup _{\{\rho(X) \leq 0\}} \inf _{\{\operatorname{Var}(a)=f\}}\langle-X, a\rangle .
$$

This relation along with the previous discussions yields that $\overline{\rho^{\#}}$ is the supremum of a family of linear lower semi-continuous functions on $\mathcal{D}_{\sigma}$. This implies that $\overline{\rho^{\#}}$ is a lower semi-continuous function on $\mathcal{D}_{\sigma}$.

On the other hand we have

$$
\begin{aligned}
\left\{Y \in \mathcal{A}_{\bar{\rho}}\right\} & =\{\bar{\rho}(Y) \leq 0\} \\
& =\left\{\sup _{a \in \mathcal{D}_{\sigma}}\left\{\left\langle-\left(\mathbb{E}\left[Y \mid \mathcal{F}_{t}\right]\right)_{t \in[0, T]}, a\right\rangle-\rho^{\#}(a)\right\} \leq 0\right\} \\
& =\left\{\mathbb{E}[-Y \operatorname{Var}(a)] \leq \rho^{\#}(a), \forall a \in \mathcal{D}_{\sigma}\right\} \\
& =\left\{\mathbb{E}[-Y f] \leq \inf _{\{\operatorname{Var}(a)=f\}} \rho^{\#}(a), \forall f \in D_{\sigma}\right\} \\
& =\left\{\mathbb{E}[-f Y] \leq \overline{\rho^{\#}}(f), \forall f \in D_{\sigma}\right\},
\end{aligned}
$$

where in the third equation we use the definition of supremum and Remark 2.3. Now let $g \in D_{\sigma}$, since $\overline{\rho^{\#}}$ is a convex lower semi continuous function and a supremum of linear functions on $\mathcal{D}_{\sigma}$, we have

$$
\begin{aligned}
(\bar{\rho})^{\#}(g) & =\sup _{\left\{Y \in \mathcal{A}_{\bar{\rho}}\right\}} \mathbb{E}[-g Y] \\
& =\sin _{\left\{\mathbb{E}[-f Y] \leq \overline{\rho^{\#}}(f), \forall f \in D_{\sigma}\right\}} \mathbb{E}[-g Y]=\overline{\rho^{\#}}(g) .
\end{aligned}
$$


REMARK 2.4. By Corollary 2.1, every coherent risk measure $\rho$ on $\mathcal{R}^{\infty}$ with the Fatou property, can be identified with a subset $\mathcal{Q}$ of $\mathcal{D}_{\sigma}$. Let $\mathcal{P}=\operatorname{Var}(\mathcal{Q}):=\{\operatorname{Var}(a)$ : $a \in \mathcal{Q}\}$. By relation (2.9) it is easy to see that for all $Y \in L^{\infty}$,

$$
\bar{\rho}(Y)=\sup _{f \in \mathcal{P}} \mathbb{E}[-f Y]
$$

3. Main results. In [10] it is shown that having the Lebesgue property for a convex risk measure with the Fatou property is equivalent to the weak compactness of lower contour sets of the conjugate function. In their proof, the author of [10] use the fact that for any uniformly integrable set $\mathcal{P} \subseteq L^{1}$ and uniformly bounded sequence $Y_{n}$ converging in probability to $Y$ we have:

$$
\sup _{f \in \mathcal{P}} \mathbb{E}\left[Y_{n} f\right] \rightarrow \sup _{f \in \mathcal{P}} \mathbb{E}[Y f] .
$$

In order to extend the Lebesgue property to bounded càdlàg process risk measures, we need to find an analog of (3.1) for the space of bounded càdlàg processes. Uniform integrability is equivalent to relative compactness in the weak topology for $L^{1}$, so we could use the $\sigma\left(\mathcal{A}^{1}, \mathcal{R}^{\infty}\right)$ relatively compact set of $\mathcal{A}^{1}$ instead. Here it is worthwhile to mention that the Dunford-Pettis Theorem, which has the same spirit, states that for any two sequences $f_{n}$ in $L^{1}$ and $Y_{n}$ in the dual space $L^{\infty}$, converging weakly to $f, Y$ respectively, the sequence $\mathbb{E}\left[f_{n} Y_{n}\right]$ converges to $\mathbb{E}[f Y]$. Therefore, knowing the $\sigma\left(\mathcal{A}^{1}, \mathcal{R}^{\infty}\right)$-compact subsets of $\mathcal{A}^{1}$ would allow us to characterize the Lebesgue property for convex risk measures on $\mathcal{R}^{\infty}$. This characterization, when restricted to $L^{\infty}$, yields the characterization in [10]. This can be carried out with the embedding $i: L^{\infty} \rightarrow \mathcal{R}^{\infty}$ defined as $i(Y)=1_{[T]} Y$. On the other hand, we find that the compactness of a set $\mathcal{Q}$ in the topology $\sigma\left(\mathcal{A}^{1}, \mathcal{R}^{\infty}\right)$ is related to the compactness of $\operatorname{Var}(\mathcal{Q})$, the variation of $\mathcal{Q}$.

In the following we first state our main result, characterizing the Lebesgue property for convex risk measures, and then give the characterization of the relatively compact subsets of $\mathcal{A}^{1}$ in $\sigma\left(\mathcal{A}^{1}, \mathcal{R}^{\infty}\right)$.

THEOREM 3.1. Let $\rho: \mathcal{R}^{\infty} \rightarrow \mathbb{R}$ be a convex risk measure with Fatou property. Then the following are equivalent:

(L1) $\rho$ has Lebesgue property.

(L2) $\rho$ is continuous from below, i.e., for every sequence $\left\{X_{n}\right\}_{n \in \mathbb{N}}$ in $\mathcal{R}^{\infty}$ such that $X_{n} \leq X$ and $\left(X_{n}-X\right)^{*} \rightarrow 0$ a.s. we have $\rho\left(X_{n}\right) \rightarrow \rho(X)$.

(L3) For all $c \in \mathbb{R},\left\{a \in \mathcal{A}^{1} ; \rho^{*}(a) \leq c\right\}$ is relatively compact in $\sigma\left(\mathcal{A}^{1}, \mathcal{R}^{\infty}\right)$.

(L4) For all $c \in \mathbb{R},\left\{a \in \mathcal{D}_{\sigma} ; \rho^{\#}(a) \leq c\right\}$ is relatively compact in $\sigma\left(\mathcal{A}^{1}, \mathcal{R}^{\infty}\right)$.

(L5) $\rho$ always attains its maximum in (2.3).

(L6) $\bar{\rho}$ has Lebesgue property.

(L7) $\bar{\rho}$ is continuous from below, i.e., for every sequence $\left\{Y_{n}\right\}_{n \in \mathbb{N}}$ in $L^{\infty}$ such that $Y_{n} \uparrow Y$ a.s. we have $\bar{\rho}\left(Y_{n}\right) \uparrow \bar{\rho}(Y)$.

(L8) For all $c \in \mathbb{R},\left\{f \in L^{1} ;(\bar{\rho})^{*}(f) \leq c\right\}$ is relatively compact in $\sigma\left(L^{1}, L^{\infty}\right)$. 
(L9) For all $c \in \mathbb{R},\left\{f \in D_{\sigma} ;(\bar{\rho})^{\#}(f) \leq c\right\}$ is relatively compact in $\sigma\left(L^{1}, L^{\infty}\right)$.

(L10) $\bar{\rho}$ always attains its maximum in (2.10).

Proof. The implications $(L 6) \Leftrightarrow(L 7) \Leftrightarrow(L 8) \Leftrightarrow(L 9) \Leftrightarrow(L 10)$ are directly conclusion of Theorem 5.2 in [10].

The implication $(L 1) \Rightarrow(L 2) \Rightarrow(L 7)$ can be easily concluded by using the definition of the Lebesgue property and the definition of $\bar{\rho}$.

$(L 8) \Rightarrow(L 3)$. Let $a \in \mathcal{A}_{+}^{1}$ be such that $\rho^{*}(a) \leq c$ for some real number $c$. By the conjugate function definition, $\forall X \in \mathcal{R}^{\infty}$ we have $-\langle X, a\rangle-\rho(X) \leq c$. In particular, this is true for every random process like $\Pi^{\mathrm{op}}(Y)=\Pi^{\mathrm{op}}\left(\mathbb{E}\left[Y \mid \mathcal{F}_{t}\right]_{t \in[0, T]}\right)$ (according to Remark 2.3), where $Y \in L^{\infty}$. By (2.9) we conclude that $-\mathbb{E}[\operatorname{Var}(a) Y]-\bar{\rho}(Y) \leq c$ for every $Y \in L^{\infty}$. Therefore, we have $\operatorname{Var}\left(\left\{a \in \mathcal{A}_{+}^{1} \mid \rho^{*}(a) \leq c\right\}\right) \subseteq\left\{\mu \in L_{+}^{1} \mid \bar{\rho}^{*}(\mu) \leq c\right\}$. By assumption $(L 6), \operatorname{Var}\left(\left\{a \in \mathcal{A}_{+}^{1} \mid \rho^{*}(a) \leq c\right\}\right)$ is relatively compact in $\sigma\left(L^{1}, L^{\infty}\right)$, hence by Theorem $3.2\left\{a \in \mathcal{A}_{+}^{1} \mid \rho^{*}(a) \leq c\right\}$ is relatively compact in $\sigma\left(\mathcal{A}^{1}, \mathcal{R}^{\infty}\right)$.

$(L 3) \Rightarrow(L 4)$ is clear.

$(L 4) \Rightarrow(L 1)$. First we assume that $\rho$ is positively homogeneous. With this assumption, for every real number $c$ the set $\left\{a \in \mathcal{D}_{\sigma} \mid \rho^{\#}(a) \leq c\right\}$ is equal to $\left\{a \in \mathcal{D}_{\sigma} \mid \rho^{\#}(a)=0\right\}$. We denote this set by $\mathcal{Q}$.

Let $X_{n}$ be a bounded sequence in $\mathcal{R}^{\infty}$ for which for some $X \in \mathcal{R}^{\infty},\left(X_{n}-X\right)^{*} \stackrel{\mathbb{P}}{\rightarrow} 0$. Since $\rho$ is positively homogeneous (therefore sub-additive) and also decreasing we have

$$
|\rho(W)-\rho(V)| \leq \rho\left(-(W-V)^{+}\right)+\rho\left(-(V-W)^{+}\right), \forall W, V \in \mathcal{R}^{\infty}
$$

This inequality allows us to assume that $X_{n} \leq 0, X=0$ and $\left(X_{n}\right)^{*} \stackrel{\mathbb{P}}{\rightarrow} 0$. Using assumption $(L 2), \mathcal{Q}$ is relatively compact in the topology $\sigma\left(\mathcal{A}^{1}, \mathcal{R}^{\infty}\right)$. Therefore, Theorem 3.2 gives that the closed convex set $\operatorname{Var}(\mathcal{Q})$ is $\sigma\left(L^{1}, L^{\infty}\right)$-compact and as a consequence by Theorem 5.1 in $[10]$ the convex function $Y \mapsto \sup _{f \in \operatorname{Var}(\mathcal{Q})} \mathbb{E}[-f Y]$ has the Lebesgue property. Hence by (2.2) we have:

$$
0 \leq \rho\left(X_{n}\right)=\sup _{a \in \mathcal{Q}}\left\langle-X_{n}, a\right\rangle \leq \sup _{f \in \operatorname{Var}(\mathcal{Q})} \mathbb{E}\left[\left(X_{n}\right)^{*} f\right] \stackrel{n}{\rightarrow} 0
$$

Now consider that the convex function $\rho$ is not necessarily positive homogeneous. Let $X_{n}$ and $X$ be bounded in $\mathcal{R}^{\infty}$ such that $\left(X_{n}-X\right)^{*} \stackrel{\mathbb{P}}{\rightarrow} 0$ (we adopt this part of the proof from the proof of Theorem $5.1[10])$. Since $X_{n}$ is uniformly bounded then there is a bounded sequence $c_{n} \in \mathbb{R}^{+}$and a positive number $\varepsilon$ such that:

$$
\rho\left(X_{n}\right) \leq \sup _{\rho^{\#}(a) \leq c_{n}}\left\langle-X_{n}, a\right\rangle-c_{n}+\varepsilon
$$

Let $c$ be a cluster point of $c_{n}$ and $I \subseteq \mathbb{N}$ such that $\left|c_{n}-c\right|<\varepsilon$ for all $n \in I$.

Let $\rho_{1}(X):=\sup _{\left\{\rho^{\#}(a) \leq c+\varepsilon\right\}}\langle-X, a\rangle$. Since $\rho_{1}$ is positively homogeneous, it has the 
Lebesgue property. Now we have

$$
\begin{aligned}
\rho(X) & \geq \sup _{\left\{\rho^{*}(\mu) \leq c+\varepsilon\right\}}\langle-X, \mu\rangle-c-\varepsilon \\
& =\rho_{1}(X)-c-\varepsilon \\
& =\lim _{n \in I} \rho_{1}\left(X_{n}\right)-c-\varepsilon \\
& \geq \lim _{n \in I} \sup _{\left\{\rho^{*}(\mu) \leq c_{n}\right\}}\left\langle-X_{n}, \mu\right\rangle-c-\varepsilon \\
& \geq \lim _{n \in I} \rho\left(X_{n}\right)-3 \varepsilon \\
& \geq \liminf \rho\left(X_{n}\right)-3 \varepsilon .
\end{aligned}
$$

Since $\varepsilon>0$ is arbitrary the proof is complete.

$(L 1) \Rightarrow(L 6)$. Let $\left(Y_{n}\right)_{n \in \mathbb{N}}$ be a uniformly bounded sequence in $L^{\infty}$ converging in probability to $Y \in L^{\infty}$. Since $\left(Y_{n}\right)_{n \in \mathbb{N}}$ is uniformly bounded, it is also uniformly integrable and consequently $Y_{n} \rightarrow Y$ in $L^{1}$. Now we have

$$
c \mathbb{P}\left(\sup _{t \leq T}\left|\mathbb{E}\left[Y_{n}-Y \mid \mathcal{F}_{t}\right]_{t \in[0, T]}\right|>c\right) \leq c \mathbb{P}\left(\sup _{t \leq T} \mathbb{E}\left[\mid Y_{n}-Y \| \mathcal{F}_{t}\right]>c\right) \leq\left\|Y_{n}-Y\right\|_{L^{1}},
$$

for all $c>0$. We used Jensen's and Doob's inequalities, in the first and second inequalities respectively. Since $Y_{n} \rightarrow Y$ in $L^{1}$, we have $X_{n} \rightarrow X$ in probability over $[0, T]$, where $X_{n}=\left(\mathbb{E}\left[Y_{n} \mid \mathcal{F}_{t}\right]\right)_{t \in[0, T]}$ and $X=\left(\mathbb{E}\left[Y \mid \mathcal{F}_{t}\right]\right)_{t \in[0, T]}$. From the Lebesgue property it turns out that $\rho\left(X_{n}\right) \rightarrow \rho(X)$, which by definition gives the Lebesgue property for $\bar{\rho}$.

$(L 3) \Rightarrow(L 5)$. Let $X \in \mathcal{R}^{\infty}$ be fixed. For every $0<\epsilon \leq 1$, by (2.2) there is an $a^{\epsilon} \in \mathcal{D}_{\sigma}$ such that $\rho(X) \leq-\left\langle X, a^{\epsilon}\right\rangle-\rho^{*}\left(a^{\epsilon}\right)+\epsilon$. Then it follows that $\rho^{*}\left(a^{\epsilon}\right) \leq-\left\langle X, a^{\epsilon}\right\rangle-\rho(X)+\epsilon \leq \operatorname{Const}(X)$, for all $0<\epsilon \leq 1$, where $\operatorname{Const}(X)$ is a real number only depending on $X$. Since $\epsilon$ can be chosen small enough, one can see that $\rho(X)=\sup _{\left\{a \in \mathcal{D}_{\sigma} \mid \rho^{*}(a) \leq \operatorname{Const}(X)\right\}}\left\{-\langle X, a\rangle-\rho^{*}(a)\right\}$. By our assumption $\left\{a \in \mathcal{D}_{\sigma} \mid \rho^{*}(a) \leq \operatorname{Const}(X)\right\}$ is compact. Now $\left\{a^{\frac{1}{n}}\right\}_{n \in \mathbb{N}}$ is a sequence in $\left\{a \in \mathcal{D}_{\sigma} \mid \rho^{*}(a) \leq \operatorname{Const}(X)\right\}$, which by compactness, has a subsequence $\left\{a^{\frac{1}{n_{k}}}\right\}$ tending to some $a \in\left\{a \in \mathcal{D}_{\sigma} \mid \rho^{*}(a) \leq \operatorname{Const}(X)\right\}$. Taking liminf of both sides of $\rho(X) \leq-\left\langle X, a^{\frac{1}{n_{k}}}\right\rangle-\rho^{*}\left(a^{\frac{1}{n}}\right)+\frac{1}{n}$, by lower semi-continuity of $\rho^{*}$ we get $\rho(X) \leq-\langle X, a\rangle-\rho^{*}(a)$. On the other hand by $(2.2)$ we have $\rho(X) \geq-\langle X, a\rangle-\rho^{*}(a)$ which implies $\rho(X)=-\langle X, a\rangle-\rho^{*}(a)$.

$(L 5) \Rightarrow(L 10)$. Fix $Y \in L^{\infty}$ and let $X=\left(\mathbb{E}\left[Y \mid \mathcal{F}_{t}\right]\right)_{t \in[0, T]}$. Then by assumption there is $a^{X} \in \mathcal{D}_{\sigma}$ in which the maximum in (2.3) is attained, i.e. $\bar{\rho}(Y)=\rho(X)=$ $-\left\langle X, a^{X}\right\rangle-\rho^{\#}\left(a^{X}\right)=\mathbb{E}\left[-Y \operatorname{Var}\left(a^{X}\right)\right]-\rho^{\#}\left(a^{X}\right)$. This implies that for every $a$ with $\operatorname{Var}(a)=\operatorname{Var}\left(a^{X}\right)$ we have $\mathbb{E}\left[-Y \operatorname{Var}\left(a^{X}\right)\right]-\rho^{\#}\left(a^{X}\right) \geq \mathbb{E}[-Y \operatorname{Var}(a)]-\rho^{\#}(a)$ and consequently $\rho^{\#}\left(a^{X}\right) \leq \rho^{*}(a), \forall a, \operatorname{Var}(a)=\operatorname{Var}\left(a^{X}\right)$. It follows that

$$
\rho^{\#}\left(a^{X}\right)=\inf _{\left\{a \in \mathcal{D}_{\sigma} \mid \operatorname{Var}(a)=\operatorname{Var}\left(a^{X}\right)\right\}} \rho^{\#}(a),
$$


which by Theorem 2.1 yields $\rho^{\#}\left(a^{X}\right)=\overline{\rho^{\#}}\left(\operatorname{Var}\left(a^{X}\right)\right)=(\bar{\rho}) \#\left(\operatorname{Var}\left(a^{X}\right)\right)$. Now it turns out that $\bar{\rho}(Y)=\mathbb{E}\left[-Y \operatorname{Var}\left(a^{X}\right)\right]-\bar{\rho}^{\#}\left(\operatorname{Var}\left(a^{X}\right)\right)$, which shows $\bar{\rho}$ attains its maximum at $\operatorname{Var}\left(a^{X}\right)$.

THEOREM 3.2. The following conditions are equivalent:

(C1) $\mathcal{Q}$ is relatively compact in $\sigma\left(\mathcal{A}^{1}, \mathcal{R}^{\infty}\right)$.

(C2) $\operatorname{Var}(\mathcal{Q})$ is relatively compact in $\sigma\left(L^{1}, L^{\infty}\right)$.

(C3) $\mathcal{Q}$ is bounded and for all $\varepsilon>0$ there exists $\eta>0$ such that for all $X \in \mathcal{R}^{\infty}$ bounded by 1 and with $\mathbb{E}\left[X^{*}\right] \leq \eta$, we have

$$
\sup _{a \in \mathcal{Q}}\langle|X|, a\rangle<\varepsilon .
$$

Proof. $(C 2) \Rightarrow(C 1)$ We define a topology on $\mathcal{R}^{\infty}$, generated by a family of semi-norms. For any weakly relatively compact subset $\mathcal{P}$ in $L^{1}$ let

$$
V(\mathcal{P}):=\left\{a \in \mathcal{A}^{1} \mid \exists f \in \mathcal{P} \text { s.t. } \operatorname{Var}(a) \leq|f|\right\}
$$

and define the associated semi-norm for $\mathcal{P}$ on $\mathcal{R}^{\infty}$ with

$$
P_{\mathcal{P}}(X)=\sup _{a \in V(\mathcal{P})}\langle X, a\rangle .
$$

This topology is compatible with the vector structure because all $V(\mathcal{P})$ 's are bounded. We denote this topology by $\sigma^{1}$. Let $\left(\mathcal{R}^{\infty}\right)^{\prime}$ be the dual of $\mathcal{R}^{\infty}$ with respect to the topology $\sigma^{1}$. It is clear that $\mathcal{A}^{1} \subseteq\left(\mathcal{R}^{\infty}\right)^{\prime}$. We want to show that $\mathcal{A}^{1}=\left(\mathcal{R}^{\infty}\right)^{\prime}$.

Let $\mu$ be an arbitrary element of $\left(\mathcal{R}^{\infty}\right)^{\prime}$ and $X_{n}$ be a non-negative sequence of uniformly bounded members in $\mathcal{R}^{\infty}$ such that $\left(X_{n}\right)^{*} \stackrel{\mathbb{P}}{\rightarrow} 0$. By $(3.1)$, we have

$$
0 \leq P_{\mathcal{P}}\left(X_{n}\right) \leq \sup _{f \in \mathcal{P}} \mathbb{E}\left[\left(X_{n}\right)^{*}|f|\right] \rightarrow 0
$$

(3.3) implies that $X_{n} \stackrel{\sigma^{1}}{\longrightarrow} 0$ and therefore $\mu\left(X_{n}\right) \rightarrow 0$. This shows that $\mu$ is finitely additive. Also from (3.3) it yields that the functional $\mu$ is order bounded (i.e., for every $\left.W, \sup _{\{U \leq W\}} \mu(U)<\infty\right)$. Since $\mathcal{R}^{\infty}$ is a Riesz space, from the general theory of Riesz spaces $\mu$ can be decomposed into the difference of its positive and negative parts (for example see Aliprantis and Burkinshaw [1], Theorem 3.3). Let $\mu^{+}$be the positive part. By definition of the positive part, for any $X \geq 0, \mu^{+}(X)=\sup _{0 \leq W \leq X} \mu(W)$. Let $X_{n}$ be a positive and decreasing sequence for which $\left(X_{n}\right)^{*} \downarrow 0$ in probability. Let $0 \leq W_{n} \leq X_{n}$ be such that $\mu^{+}\left(X_{n}\right) \leq \mu\left(W_{n}\right)+\frac{1}{n}$. Since $\left(W_{n}\right)^{*} \stackrel{\mathbb{P}}{\rightarrow} 0$, by (3.3) we get that

$$
0 \leq \mu^{+}\left(X_{n}\right) \leq \mu\left(W_{n}\right)+\frac{1}{n} \rightarrow 0
$$

Given Theorem 2 of chapter VII in [7], one deduces that $\mu^{+} \in \mathcal{A}^{1}$. Similarly $\mu^{-} \in \mathcal{A}^{1}$ and therefore $\mu \in \mathcal{A}^{1}$. This completes the proof that $\mathcal{A}^{1}=\left(\mathcal{R}^{\infty}\right)^{\prime}$. 
The Corollary to Mackey's Theorem 9, section 13, chapter 2 [9] leads us to $\sigma^{1} \subseteq$ $\tau\left(\mathcal{R}^{\infty}, \mathcal{A}^{1}\right)$, where $\tau\left(\mathcal{R}^{\infty}, \mathcal{A}^{1}\right)$ is the Mackey topology. Just to recall, Mackey's topology is a topology generated with a basis of open sets around the origin defined as

$$
\left\{X \in \mathcal{R}^{\infty} \mid \sup _{C}\langle X, a\rangle<1\right\}
$$

for all $\sigma\left(\mathcal{A}^{1}, \mathcal{R}^{\infty}\right)$-compact subsets $C \subseteq \mathcal{A}^{1}$.

Let $\mathcal{P}$ be a $\sigma\left(L^{1}, L^{\infty}\right)$ relatively compact subset of $L^{1}$. By definition, the set $\left\{X \mid P_{\mathcal{P}}(X)<1\right\}$ is an open set in $\sigma^{1}$. Since $\sigma^{1} \subseteq \tau$, this set is also an open set in $\tau$. Therefore, there exists a $\sigma\left(\mathcal{A}^{1}, \mathcal{R}^{\infty}\right)$-compact set $C$ such that $\left\{X \mid \sup _{C}\langle X, a\rangle<1\right\} \subseteq\left\{X \mid P_{\mathcal{P}}(X)<1\right\}$. By polarity (which is decreasing with respect to inclusion) we have that $\left\{X \mid P_{\mathcal{P}}(X) \leq 1\right\}^{\circ} \subseteq\left\{X \mid P_{\mathcal{P}}(X)<1\right\}^{\circ} \subseteq$ $\left\{X \mid \sup _{a \in C}\langle X, a\rangle<1\right\}^{\circ}$. From the generalized Bourbaki-Alaoglu Theorem we know that the polar set of every open set in $\left(\mathcal{R}^{\infty}, \sigma^{1}\right)$, which we know has $\mathcal{A}^{1}$ as its dual, is $\sigma\left(\mathcal{A}^{1}, \mathcal{R}^{\infty}\right)$-compact. Therefore, $\left\{X \mid \sup _{C}\langle X, a\rangle<1\right\}^{\circ}$ is $\sigma\left(\mathcal{A}^{1}, \mathcal{R}^{\infty}\right)$-compact. Since $\left\{X \mid P_{\mathcal{P}}(X) \leq 1\right\}^{\circ} \subseteq\left\{X \mid \sup _{C}\langle X, a\rangle<1\right\}^{\circ}$ then $\left\{X \mid P_{\mathcal{P}}(X) \leq 1\right\}^{\circ}$ is $\sigma\left(\mathcal{A}^{1}, \mathcal{R}^{\infty}\right)$ relatively compact. By definition of polarity it is clear that $V(\mathcal{P}) \subseteq\left\{X \mid P_{\mathcal{P}}(X) \leq 1\right\}^{\circ}$, which yields that $V(\mathcal{P})$ is $\sigma\left(\mathcal{A}^{1}, \mathcal{R}^{\infty}\right)$-relatively compact. Now let $\mathcal{P}=\operatorname{Var}(\mathcal{Q})$, since $\mathcal{Q} \subseteq V(\operatorname{Var}(\mathcal{Q}))$, one concludes that $\mathcal{Q}$ is $\sigma\left(\mathcal{A}^{1}, \mathcal{R}^{\infty}\right)$-relatively compact.

$(C 1) \Rightarrow(C 3)$. Let

$$
G=\left\{X \in \mathcal{R}^{\infty} \mid \mathbb{E}\left[X^{*}\right] \leq 1, X \text { is bounded by } 1\right\} .
$$

Thus, $G$ is a bounded set in the topology $\sigma\left(\mathcal{R}^{\infty}, \mathcal{A}^{1}\right)$. Indeed, this is true since for every $a \in \mathcal{A}^{1}$ we have $|\langle X, a\rangle| \leq \mathbb{E}\left[X^{*}\right] \operatorname{Var}(a) \leq \operatorname{Var}(a)$. This implies that for every relatively compact subset $\mathcal{Q}$ in $\sigma\left(\mathcal{A}^{1}, \mathcal{R}^{\infty}\right)$,

$$
\sup _{X \in G}\left(\sup _{a \in \mathcal{Q}}|\langle X, a\rangle|\right)=: L<\infty
$$

Indeed, $X \mapsto \sup _{a \in \mathcal{Q}}\langle X, a\rangle$ is a semi-norm from which the Mackey topology is generated. Since by Mackey's Theorem 9, section 13, chapter 2, [9], $\tau\left(\mathcal{A}^{1}, \mathcal{R}^{\infty}\right)$ has the same dual as $\sigma\left(\mathcal{A}^{1}, \mathcal{R}^{\infty}\right), G$ is also bounded in $\tau$, which implies (3.4).

Now let $\eta=\frac{\epsilon}{L}$.

$(C 3) \Rightarrow(C 2)$ Let $X_{U}=\Pi^{\mathrm{op}}\left(1_{U}\right)$ where $U$ is a measurable set such that $\mathbb{P}(U)<\eta$. For a given $a \in \mathcal{Q}$, let $U^{ \pm}=U \cap\left\{a_{T}^{ \pm}-a_{0}^{ \pm}>0\right\}$. We have:

$$
\mathbb{E}\left[ \pm 1_{U^{ \pm}}\left(a_{T}^{ \pm}-a_{0}^{ \pm}\right)\right]=\left\langle\left|X_{U^{ \pm}}\right|, a\right\rangle<\varepsilon,
$$

which shows that $\operatorname{Var}\left(\mathcal{Q}_{ \pm}\right)$and consequently $\operatorname{Var}(\mathcal{Q})$ are uniformly integrable. $\square$ The following corollary is an immediate consequence of Theorem 3.2.

Corollary 3.1. $\mathcal{Q} \subseteq \mathcal{A}^{1}$ is $\sigma\left(\mathcal{A}^{1}, \mathcal{R}^{\infty}\right)$ - relatively compact if and only if $\operatorname{Var}(\mathcal{Q})$ is uniformly integrable. 
REMARK 3.1. It is not very difficult to see that statements $(C 1)$ and $(C 2)$ are also equivalent for duality $\left(\mathcal{R}^{p}, \mathcal{A}^{q}\right)$ when $1 \leq p<\infty$ and $\frac{1}{p}+\frac{1}{q}=1$. Indeed it is a straightforward application of Banach-Alaoglu theorem given the fact that $\hat{\mathcal{A}}^{q}$ is the topological dual of $\hat{\mathcal{R}}^{p}$ for $p \neq 1$ (see Theorems 65, 67 of section VII, [7]) and that $\Pi^{*}$ is a continuous mapping from $\hat{\mathcal{A}}^{q}$ to $\mathcal{A}^{q}$.

We complete the section by stating a result which is a form of James' Theorem for the duality $\left(\mathcal{A}^{q}, \mathcal{R}^{p}\right)$. Indeed, for the case $1 \leq p<\infty$ following the same idea as in Remark 3.1, one comes up with the result. However, for the case $p=\infty$ we need the following theorem

TheOREM 3.3 (James' Theorem for $\left(\mathcal{A}^{1}, \mathcal{R}^{\infty}\right)$ ). Let $\mathcal{Q} \subseteq \mathcal{A}_{+}^{1}$ be a convex, $\sigma\left(\mathcal{A}^{1}, \mathcal{R}^{\infty}\right)$-closed subset of $\mathcal{A}^{1}$. The set $\mathcal{Q}$ is compact in $\sigma\left(\mathcal{A}^{1}, \mathcal{R}^{\infty}\right)$ if and only if for each member $X \in \mathcal{R}^{\infty}$ it attains its supremum on $\mathcal{Q}$.

Proof. $(\Rightarrow)$ Is clear.

$(\Leftarrow)$ Define the convex function $\rho$ by:

$$
\rho(X):=\sup _{a \in \mathcal{Q}}\langle X, a\rangle .
$$

It is not difficult to see that $\operatorname{Var}(\mathcal{Q})$ is convex and weakly closed. Let $Y \in L^{\infty}$. It is easy to see that $\bar{\rho}(Y)=\sup _{\mathbb{E}} \mathbb{E}[Y f]$. By assumption, for any $Y \in L^{\infty}$ there exists an $a \in \mathcal{Q}$ such that

$$
\rho\left(\left(\mathbb{E}\left[Y \mid \mathcal{F}_{t}\right]\right)_{0 \leq t \leq T}\right)=\left\langle\left(\mathbb{E}\left[Y \mid \mathcal{F}_{t}\right]\right)_{0 \leq t \leq T}, a\right\rangle .
$$

This gives $\bar{\rho}(Y)=\mathbb{E}[\operatorname{Var}(a) Y]$. This fact with James' Theorem implies that $\operatorname{Var}(\mathcal{Q})$ is weakly compact. Now by Theorem 3.2 we deduce that $\mathcal{Q}$ is compact in $\sigma\left(\mathcal{A}^{1}, \mathcal{R}^{\infty}\right)$. $\square$

4. Examples of risk measures with Lebesgue property. In this section we present the first series of examples. In the sequel we will see how our results can help to figure out whether a convex risk measure has the Lebesgue property or not.

Before giving examples, we show how the Lebesgue property can be used in order to approximate the risk. Let $X$ be a random process in $\mathcal{R}^{\infty}$. A natural way of approximating this process within a time discretization is to construct the following sequence

$$
X_{n}=\sum_{i=0}^{2^{n}-1} 1_{\left[\frac{i}{2^{n}} T, \frac{i+1}{2^{n}} T\right)} X_{\frac{i}{2^{n}} T}+X_{T}
$$

It is clear that since $X$ is a càdlàg process, $\left(X_{n}-X\right)^{*}$ converges to zero in probability. Now, for a convex risk measure $\rho$ with Lebesgue property we have that $\rho\left(X_{n}\right) \rightarrow \rho(X)$. This is no longer true if we only know that the risk measure has the Fatou property. Actually having the Fatou property, we can only say that $\rho\left(X_{n}\right)$ converges to $\rho(X)$ if $X_{n}$ decreases to $X$. On the other hand, as a decreasing sequence, we cannot choose

$$
X_{n}^{\prime}=\sum_{i=0}^{2^{n}-1} 1_{\left[\frac{i}{2^{n}} T, \frac{i+1}{2^{n}} T\right)} \sup _{\left[\frac{i}{2^{n}} T, \frac{i+1}{2^{n}} T\right)} X_{t}+X_{T},
$$

which is no longer adapted. As one can see, the Lebesgue property is a very strong assumption in approximating risk. In what follows, interestingly we will see that many important examples have the Lebesgue property which enables us to approximate. 
In the following discussions, the first two examples are taken from [5]. The third one is the Snell envelope of a random process which is used in pricing an American option. In the fourth example we introduce for the first time a Cumulative-Stopping risk measure. This risk measure, besides having a very natural structure, provides us an exact formula for allocation when we deal with an $\alpha$-stable random process.

In the sequel $\mathcal{P}_{\sigma}$ is a subset of $D_{\sigma}=\left\{f \in L_{+}^{1} \mid \mathbb{E}[f]=1\right\}$. The coherent risk measure $\rho_{\sigma}$ is defined on $L^{p}$ as follows

$$
\rho_{\sigma}(X):=\sup _{f \in \mathcal{P}_{\sigma}} \mathbb{E}[-f X] .
$$

Example. Let $\Theta$ be a set of stopping times and $\rho$ be defined as follows

$$
\rho(Y)=\sup _{a \in \mathcal{Q}_{\Theta}}\langle-Y, a\rangle,
$$

where $\mathcal{Q}_{\Theta}=\left\{\left(0, \mathbb{E}\left[f \mid \mathcal{F}_{\theta}\right] 1_{t \geq \theta}\right) \mid f \in \mathcal{P}_{\sigma}, \theta \in \Theta\right\}$.

For example, $\Theta$ can be a ruin time or the time that insurance surplus hits a specific barrier (see for instance [3]). Also, $\Theta$ can be the set of exercising times of an American option.

It is easy to see that

$$
\rho(X)=\sup _{\theta \in \Theta} \rho_{\sigma}\left(X_{\theta}\right), \forall X \in \mathcal{R}^{p} .
$$

By Remark 2.4, the static risk is calculated as

$$
\begin{aligned}
\bar{\rho}(Y) & =\sup _{a \in \mathcal{Q}}\left\langle-\left(\mathbb{E}\left[Y \mid \mathcal{F}_{t}\right]\right)_{t \in[0, T]}, a\right\rangle \\
& =\sup _{f \in \mathcal{P}_{\sigma}, \theta \in \Theta} \mathbb{E}\left[\mathbb{E}\left[-Y \mid \mathcal{F}_{\theta}\right] f\right] \\
& =\sup _{f \in \mathcal{P}_{\sigma}, \theta \in \Theta} \mathbb{E}\left[-Y \mathbb{E}\left[f \mid \mathcal{F}_{\theta}\right]\right] .
\end{aligned}
$$

According to Theorem 3.1, $\bar{\rho}$ has the Lebesgue property iff

$$
\left\{\mathbb{E}\left[f \mid \mathcal{F}_{\theta}\right] \mid \theta \in \Theta, f \in \mathcal{P}_{\sigma}\right\}
$$

is uniformly integrable. Therefore, by Theorem 3.1, $\rho$ has Lebesgue property iff the above set is uniformly integrable. In particular, it has Lebesgue property when $\mathcal{P}_{\sigma}$ is uniformly integrable. In other words, $\rho$ has Lebesgue property if $\rho_{\sigma}$ does.

Example. For any random variable $f \in \mathcal{P}_{\sigma} \subseteq D_{\sigma}$ and stopping time $\theta \in \Theta$, define the random process $f_{\theta}$ as follows:

$$
f_{\theta}(t)= \begin{cases}\frac{t}{\theta} \mathbb{E}\left[f \mid \mathcal{F}_{t}\right] & t \leq \theta, \\ \mathbb{E}\left[f \mid \mathcal{F}_{\theta}\right] & \text { otherwise. }\end{cases}
$$

Then, on $\mathcal{R}^{\infty}$, let

$$
\rho(X)=\sup _{a \in \mathcal{Q}}\langle-X, a\rangle
$$


where $\mathcal{Q}=\left\{\left(f_{\theta}, 0\right) \mid f \in \mathcal{P}_{\sigma}, \theta \in \Theta\right\}$. It is easy to see that

$$
\begin{aligned}
& \rho(X)=\sup _{\theta \in \Theta} \rho_{\sigma}\left(\frac{1}{\theta} \int_{0}^{\theta} X_{t} d t\right), \\
& \operatorname{Var}(\mathcal{Q})=\left\{\mathbb{E}\left[f \mid \mathcal{F}_{\theta}\right] \mid f \in \mathcal{P}_{\sigma}, \theta \in \Theta\right\}, \\
& \bar{\rho}(Y)=\sup _{f \in \mathcal{P}_{\sigma}, \theta \in \Theta} \mathbb{E}\left[-Y \mathbb{E}\left[f \mid \mathcal{F}_{\theta}\right]\right], \text { for } Y \in L^{\infty} .
\end{aligned}
$$

By part (C2) of Theorem 3.2 and (L3) of Theorem 3.1, $\rho$ has Lebesgue property iff

$$
\operatorname{Var}(\mathcal{Q})=\left\{\mathbb{E}\left[f \mid \mathcal{F}_{\theta}\right] \mid f \in \mathcal{P}_{\sigma}, \theta \in \Theta\right\}
$$

is uniformly integrable. Also it has Lebesgue property if $\mathcal{P}_{\sigma}$ is uniformly integrable

Snell envelope and American option price stability. Let $X \in \mathcal{R}^{\infty}$ and $S \leq T$ be a stopping time. Let

$$
\Theta_{S}=\{\theta \geq S \mid \theta \text { is }[0, T] \text {-value stopping time }\} .
$$

Set

$$
\begin{aligned}
\rho_{S}(X) & =\operatorname{ess} \sup _{a \in \mathcal{Q}_{S}}\langle-X, a\rangle \\
& =\operatorname{ess} \sup \left\{\mathbb{E}\left[-X_{\theta} \mid \mathcal{F}_{S}\right] \mid \theta \in \Theta_{S}\right\} .
\end{aligned}
$$

The process $\rho_{t}(X)$ is the smallest super-martingale larger than $-X$ which is called the Snell envelope of $-X$, see, e.g., [5].

Now for any measurable set $A \in \mathcal{F}_{S}$ define

$$
\rho_{S}^{A}(X)=\mathbb{E}\left[\rho_{S}(X) 1_{A}\right] .
$$

It is exactly equivalent to put $\mathcal{P}_{\sigma}=\left\{\frac{1}{\mathbb{P}(A)} 1_{A}\right\}$ and $\Theta=\Theta_{S}$ in Example 1. From Example 1 we know that $\rho_{S}^{A}$ has the Lebesgue property. Since the choice of $A \in \mathcal{F}_{S}$ is arbitrary, then by (4.6), we have that for each stopping time $S$ the Snell envelope $\rho_{S}(X)$ is continuous in the weak star topology. In particular, setting $\rho_{t}=\rho_{t}^{\Omega}$, then $\rho_{t}\left(X_{n}\right) \rightarrow \rho_{t}(X)$ when $\left(X_{n}-X\right)^{*} \rightarrow 0$. This shows how one can approximate the price of an American option in continuous time by time discretization.

Cumulative-stopping risk. Let $\rho_{\sigma}$ be a risk measure on $L^{\infty}$. A natural way to assess the risk of a random process is the average of the risk over the time interval, i.e. $\frac{1}{T} \int_{0}^{T} \rho_{\sigma}\left(X_{s}\right) d s$. On the other hand, let us suppose that there exists a stopping time (or a general random time) which shows some crucial moments, important for the risk user. Then a way to measure the risk of a random process $X$ in $\mathcal{R}^{\infty}$ is to calculate

$$
\rho(X)=\int_{0}^{T} \rho_{\sigma}\left(X_{s}\right) f_{\theta}(s) d s
$$


where $f_{\theta}$ is the density function of $\theta$. This new convex risk measure is called the Cumulative-Stopping risk.

In fact, for any measure $\mu$ on $[0, T]$,

$$
\int_{0}^{T} \rho_{\sigma}\left(X_{s}\right) \mu(d s)
$$

will work and it is a mixture risk measure.

It is not very difficult to see that when the risk measure $\rho_{\sigma}$ has the Fatou property then also does $\rho$. It means that when $\rho_{\sigma}$ has a representation like (2.10) then $\rho$ has a representation like (2.2) (with $\mathcal{D}_{\sigma} \cap \mathcal{A}^{1}$ instead of $\mathcal{D}_{\sigma}$ ). On the other hand, the convex risk $\rho$ has the Lebesgue property iff $\rho_{\sigma}$ does. Actually this follows from part (L5) of Theorem 3.1.

Acknowledgment. The author would like to thank Institut de Finance Mathématique de Montréal (IFM2) for its financial support during completion of this work. Also the author thanks anonymous referees for their many good suggestions.

\section{REFERENCES}

[1] C. D. Aliprantis and O. Burkinshaw, Locally solid Riesz spaces, Academic Press [Harcourt Brace Jovanovich Publishers], New York, 1978. Pure and Applied Mathematics, Vol. 76.

[2] P. Artzner, F. Delbaen, J.-M. Eber, and D. Heath, Coherent measures of risk, Math. Finance, 1999.

[3] S. Asmussen, Ruin probabilities, volume 2 of Advanced Series on Statistical Science 85 Applied Probability, World Scientific Publishing Co. Inc., River Edge, NJ, 2000.

[4] P. Cheridito, F. Delbaen, And M. Kupper, Coherent and convex monetary risk measures for bounded càdlàg processes, Stochastic Process. Appl., 112:1 (2004), pp. 1-22.

[5] P. Cheridito, F. Delbaen, And M. Kupper, Coherent and convex monetary risk measures for unbounded càdlàg processes, Finance Stoch., 9:3 (2005), pp. 369-387.

[6] F. Delbaen, Coherent risk measures on general probability spaces, In Advances in finance and stochastics, pp. 1-37. Springer, Berlin, 2002.

[7] C. Dellacherie and P.-A. Meyer, Probabilités et potentiel. Chapitres $V$ à VIII, volume 1385 of "Actualités Scientifiques et Industrielles [Current Scientific and Industrial Topics]", Hermann, Paris, revised edition, 1980. Théorie des martingales. [Martingale theory].

[8] H. Föllmer AND A. SChied, Robust preferences and convex measures of risk, in "Advances in finance and stochastics", pp. 39-56. Springer, Berlin, 2002.

[9] A. Grothendieck, Topological vector spaces, Gordon and Breach Science Publishers, New York, 1973. Translated from the French by Orlando Chaljub, Notes on Mathematics and its Applications.

[10] E. Jouini, W. Schachermayer, And N. Touzi, Law invariant risk measures have the Fatou property, In "Advances in mathematical economics. Vol. 9", volume 9 of Adv. Math. Econ., pp. 49-71. Springer, Tokyo, 2006. 
H. ASSA 